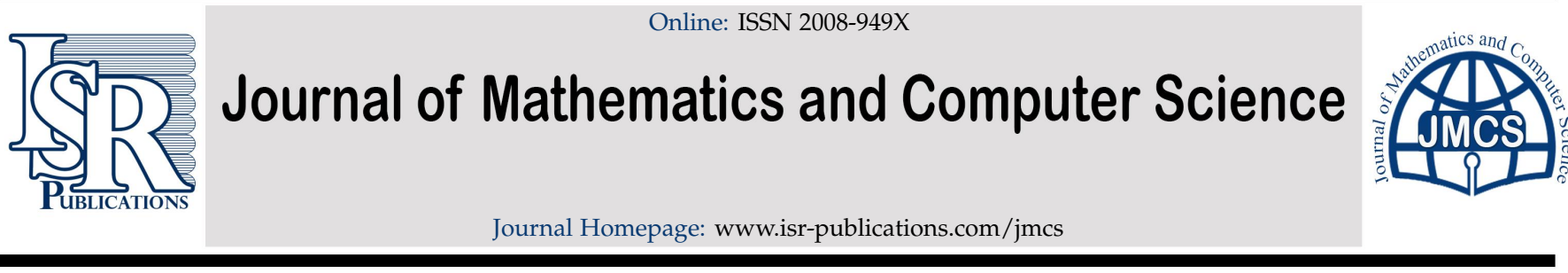

\title{
A class of second order nondifferentiable symmetric duality relations under generalized assumptions
}

\author{
Ramu Dubey ${ }^{\mathrm{a}}$, Vandana $^{\mathrm{b}, *}$, Vishnu Narayan Mishra $^{\mathrm{c}}$, Seda Karateke ${ }^{\mathrm{d}}$ \\ ${ }^{a}$ Department of Mathematics, J.C. Bose University of Science and Technology, YMCA, Faridabad-121 006, India. \\ ${ }^{b}$ Department of Management Studies, Indian Institute of Technology Madras, Chennai-600036, Tamil Nadu, India. \\ ${ }^{c}$ Department of Mathematics, Indira Gandhi National Tribal University, Lalpur, Amarkantak 484 887, Madhya Pradesh, India. \\ ${ }^{d}$ Department of Mathematics and Computer Science, Faculty of Science and Letters, Istanbul Arel University, Istanbul-34537, Turkey.
}

\begin{abstract}
In this article, a pair of second-order nondifferentiable symmetric dual model in optimization problem is formulated over arbitrary cones. For a differentiable function, we consider the definition of strongly K-pseudobonvexity convexity. Next, we derive the appropriate duality results under aforesaid assumptions.
\end{abstract}

Keywords: Symmetric duality, second-order, non-differentiable, strongly K-pseudobonvexity.

2020 MSC: 90C26, 90C30, 90C $32,90 \mathrm{C} 46$.

(C)2020 All rights reserved.

\section{Introduction}

In the recent years, the analysis of multiobjective optimization problems has been a focal issue. Such multiobjective optimization problems are useful mathematical models for the investigation of real-world problems. Also many researchers motivated by works of the concept of second-order duality in nonlinear problems, introduced by Mangasarian [10] have also worked in this area. The advantage of second-order duality is considered over first-order as it gives more closer bounds. Hanson [8] in his study has cited one example which demonstrates the application of second-order duality in somewhat different perspective.

In the last several years, several optimality and duality results have been obtained for multiobjective fractional programming problems. Multiobjective fractional problem and its duality relations have been assumed under higher-order $(f, \alpha, \rho, d)$-convexity by Chen in [1]. Later on in [13], Suneja et al. derived the higher-order Mond-Weir and Schaible type nondifferentiable dual programs and their duality theorems under higher-order $(f, \rho, \sigma)$-type I-assumptions. Ying [15] studied higher-order multiobjective symmetric fractional problem and formulated its Mond-Weir type dual and duality theorems were proved under

\footnotetext{
*Corresponding author

Email addresses: rdubeyjiya@gmail.com (Ramu Dubey), vdrai1988@gmail.com (Vandana), vishnunarayanmishra@gmail.com (Vishnu Narayan Mishra), sedakarateke@arel.edu.tr (Seda Karateke)
}

doi: $10.22436 /$ jmcs.021.02.03

Received: 2019-06-17 Revised: 2020-01-27 Accepted: 2020-02-10 
higher-order $(f, \alpha, \rho, d)$-convexity assumptions. Many researchers have also worked in the same directions (see $[3-6,9,11,14])$.

The purpose of the present work is to study second order symmetric duality over arbitrary cones for nondifferentiable type programs under strongly K-pseudobonvexity assumptions. The paper is organized as follows. In Section 2, we present some relevant and basic preliminaries. In Section 3, we formulate a nondifferentiable second order symmetric dual problem with cone constraints and derive appropriate duality results under strongly K-pseodobonvexity assumptions.

\section{Preliminaries and definitions}

Definition 2.1 ([2]). Let $C$ be a compact convex set in $R^{n}$. The support function of $C$ is defined by

$$
s(x \mid C)=\max \left\{x^{\top} y: y \in C\right\} .
$$

A support function, being convex and everywhere finite, has a subdifferential, that is, there exists a $z \in R^{n}$ such that $s(y \mid C) \geqslant s(x \mid C)+z^{\top}(y-x)$ for every $x \in C$. The subdifferential of $s(x \mid C)$ is given by

$$
\partial s(x \mid C)=\left\{z \in C: z^{\top} x=s(x \mid C)\right\} .
$$

For a convex set $\mathrm{D} \subset \mathrm{R}^{\mathrm{n}}$, the normal cone to $\mathrm{D}$ at a point $x \in \mathrm{D}$ is defined by

$$
N_{D}(x)=\left\{y \in R^{n}: y^{\top}(z-x) \leqq 0, \text { for all } z \in D\right\}
$$

When $C$ is a compact convex set, $y \in N_{C}(x)$ if and only if $s(y \mid C)=x^{\top} y$, or equivalently, $x \in \partial s(y \mid C)$.

Let $X \subset R^{n}$ and $K \subset R^{k}$ be a closed convex pointed cone with intK $\neq \phi$. Now, we consider the definitions of K-pseudoinvex and K-pseudobonvex functions with respect to $\eta$.

Definition 2.2. A differentiable function $f: X \rightarrow R^{k}$ is K-pseudoinvex at $u \in X$ with respect to $\eta: X \times X \longrightarrow$ $R^{n}$, if for every $(x, p) \in X \times R^{n}$,

$$
-\eta^{\top}(x, u)\left[\nabla_{u} f(u)\right] \notin \operatorname{int}(K) \Longrightarrow-[f(x)-f(u)] \notin \operatorname{int}(K) .
$$

Definition 2.3. A differentiable function $f: X \rightarrow R^{k}$ is strongly $K$-pseudoinvex at $u \in X$ with respect to $\eta: X \times X \longrightarrow R^{n}$, if for every $(x, p) \in X \times R^{n}$,

$$
-\eta^{\top}(x, u)\left[\nabla_{u} f(u)\right] \notin \operatorname{int}(K) \Longrightarrow[f(x)-f(u)] \in K .
$$

Definition 2.4. A differentiable function $f: X \rightarrow R^{k}$ is $K$-pseudobonvex at $u \in X$ with respect to $\eta$ : $X \times X \longrightarrow R^{n}$, if for every $(x, p) \in X \times R^{n}$,

$$
\left.-\eta^{\top}(x, u)\left[\nabla_{u} f(u)+\nabla_{u u} f(u)\right\} p\right] \notin \operatorname{int}(K) \Longrightarrow-\left[f(x)-f(u)+\frac{1}{2} p^{\top} \nabla_{u u} f(u) p\right] \notin \operatorname{int}(K) .
$$

Definition 2.5. A differentiable function $f: X \rightarrow R^{k}$ is strongly $K$-pseudobonvex at $u \in X$ with respect to $\eta: X \times X \longrightarrow R^{n}$, if for every $(x, p) \in X \times R^{n}$,

$$
-\eta^{\top}(x, u)\left[\nabla_{u} f(u)+\nabla_{u u} f(u) p\right] \notin \operatorname{int}(K) \Longrightarrow\left[f(x)-f(u)+\frac{1}{2} p^{\top} \nabla_{u u} f(u) p\right] \in K .
$$

Remark 2.6. $\frac{1}{2} p^{\top} \nabla_{u u} f(u) p$ represents the vector of components

$$
\left.\left(\frac{1}{2} p^{\top} \nabla_{u u} f_{1}(u) p, \frac{1}{2} p^{\top} \nabla_{u u} f_{2}(u) p\right), \ldots, \frac{1}{2} p^{\top} \nabla_{u u} f_{k}(u) p\right)
$$


Remark 2.7. For proving the strong and converse duality theorems, we need necessary conditions proposed by Suneja et al. in [12]. They considered the the following multiobjective programming problem:

(VP) K-Minimize $f(x)$,

subject to $X_{0}=\left\{x \in R^{n}:-g(x) \in Q, x \in C\right\}$,

where $f: R^{n} \rightarrow R^{k}, g: R^{n} \rightarrow R^{m}, C \subset R^{n}, K$ and $Q$ are closed convex cones with nonempty interiors in $R^{k}$ and $R^{m}$, respectively.

Definition 2.8. A point $u \in X_{0}$ is said to be a weak minimum of (VP), if there exists no other $x \in X_{0}$ such that $f(u)-f(x) \in \operatorname{int}(K)$.

Definition 2.9. The positive polar cone $K^{*}$ of $K$ is defined as follows:

$$
\mathrm{K}^{*}=\left\{z \in \mathrm{R}^{\mathrm{p}}: \mathrm{x}^{\top} z \geqslant 0 \text {, for all } \mathrm{x} \in \mathrm{K}\right\} .
$$

Lemma 2.10. If $\mathrm{u} \in \mathrm{X}_{0}$ is a weak minimum of $(V P)$, then there exists $\bar{\alpha} \in \mathrm{K}^{*}$ and $\beta \in \mathrm{Q}^{*}$ not both zero such that

$$
\begin{gathered}
\left(\bar{\alpha}^{\top} \nabla \mathbf{f}(\mathfrak{u})+\bar{\beta}^{\top} \nabla \mathrm{g}(\mathfrak{u})\right)^{\top}(x-\mathfrak{u}) \geqslant 0, \quad \forall x \in \mathrm{C}, \\
\bar{\beta}^{\top} \nabla \mathrm{g}(\mathfrak{u})=0 .
\end{gathered}
$$

\section{Second-order nondifferentiable symmetric duality model}

In this section, we formulate the following pair of second-order nondifferentiable symmetric dual programs over arbitrary constraints:

$$
\begin{aligned}
\text { (NSOP)K-Minimize } & W(x, y, r, p)=f(x, y)+s(x \mid B)-y^{\top} r-\frac{1}{2} p^{\top} \nabla_{y y} f(x, y) p, \\
\text { subject to } \quad & \left.-\sum_{i=1}^{k} \lambda_{i}\left[\nabla_{y} f_{i}(x, y)-r_{i}+\nabla_{y y} f_{i}(x, y)\right\} p\right] \in C_{2}^{*} \\
& y^{\top}\left[\sum_{i=1}^{k} \lambda_{i}\left(\nabla_{y} f_{i}(x, y)-r_{i}+\nabla_{y y} f_{i}(x, y) p\right)\right] \geqslant 0 \\
& p^{\top}\left[\sum_{i=1}^{k} \lambda_{i}\left(\nabla_{y} f_{i}(x, y)-r_{i}+\nabla_{y y} f_{i}(x, y) p\right)\right] \geqslant 0 \\
& x \in C_{1}, r \in F, \lambda \in \operatorname{int}\left(K^{*}\right)
\end{aligned}
$$

(NSOD) K-Maximize $T(u, v, t, q)=f(u, v)-s(v \mid F)+u^{\top} t-\frac{1}{2} q^{\top} \nabla_{u u} f(u, v) q$,

$$
\begin{array}{ll}
\text { subject to } & \sum_{i=1}^{k} \lambda_{i}\left[\nabla_{\mathfrak{u}} f_{i}(u, v)+t_{i}+\nabla_{u u} f_{i}(u, v) q\right] \in C_{1}^{*}, \\
& u^{\top}\left[\sum_{i=1}^{k} \lambda_{i}\left(\nabla_{u} f_{i}(u, v)+t+\nabla_{u u} f_{i}(u, v) q\right)\right] \leqslant 0, \\
& q^{\top}\left[\sum_{i=1}^{k} \lambda_{i}\left(\nabla_{u} f_{i}(u, v)+t+\nabla_{u u} f_{i}(u, v) q\right)\right] \leqslant 0, \\
& v \in C_{2}, t \in B, \lambda \in \operatorname{int}\left(K^{*}\right),
\end{array}
$$

where $C_{1}$ and $C_{2}$ are closed convex cones with non-empty interiors in $R^{n}$ and $R^{m}$, respectively. $C_{1}^{*}$ and $C_{2}^{*}$ are positive polar cones of $C_{1}$ and $C_{2}$, respectively, and $f: R^{n} \times R^{m} \rightarrow R^{k}$ is differentiable function.

Let $R^{0}$ and $S^{0}$ be feasible solution of (NSOP) and (NSOD), respectively. 
Theorem 3.1 (Weak duality theorem). Let $(x, y, \lambda, r, p) \in R^{0}$ and $(u, v, \lambda, t, q) \in S^{0}$. Let

(i) $\mathrm{f}(., v)$ be strongly K-pseudobonvex and $(.)^{\top} \mathrm{t}$ be strongly $\mathrm{K}$ - psedoinvex at $\mathrm{u}$ with respect to $\eta$;

(ii) $-\mathrm{f}(\mathrm{x},$.$) be \mathrm{K}-$ pseudobonvex and $(.)^{\mathrm{T}} \mathrm{r}$ be $\mathrm{K}$ - psedoinvex at $\mathrm{y}$ with respect to $\xi$;

(iii) $\eta(x, u)+u+q \in C_{1}$ for all $x \in C_{1}$;

(iv) $\xi(v, y)+y+p \in C_{2}$ for all $y \in C_{2}$.

Then

$$
T(u, v, \lambda, t, q)-W(x, y, \lambda, r, p) \notin \operatorname{int}(K)
$$

Proof. Suppose, on contrary

$$
T(u, v, \lambda, t, q)-W(x, y, \lambda, r, p) \in \operatorname{int}(K)
$$

i.e.,

$$
\begin{aligned}
& \left\{f(u, v)-s(v \mid F)+u^{\top} t-\frac{1}{2} q^{\top} \nabla_{u u} f(u, v) q\right. \\
& \left.\quad-\left(f(x, y)+s(x \mid B)-y^{\top} r-\frac{1}{2} p^{\top} \nabla_{y y} f(x, y) p\right)\right\} \in \operatorname{int}(K) .
\end{aligned}
$$

From, hypothesis (iii) and (3.1), we obtain

$$
\begin{aligned}
& (\eta(x, u)+u+q)^{\top}\left[\sum_{i=1}^{k} \lambda_{i}\left(\nabla_{u} f_{i}(u, v)+t_{i}+\nabla_{u u} f_{i}(u, v) q\right)\right] \geqslant 0, \\
& \eta^{\top}(x, u)\left[\sum_{i=1}^{k} \lambda_{i}\left(\nabla_{u} f_{i}(u, v)+t_{i}+\nabla_{u u} f_{i}(u, v) q\right)\right] \geqslant-(u+q)\left[\sum_{i=1}^{k} \lambda_{i}\left(\nabla_{u} f_{i}(u, v)+t_{i}+\nabla_{u u} f_{i}(u, v) q\right)\right]
\end{aligned}
$$

which on using (3.2) and (3.3), it yields

$$
\eta^{\top}(x, u)\left[\sum_{i=1}^{k} \lambda_{i}\left(\nabla_{u} f_{i}(u, v)+t_{i}+\nabla_{u u} f_{i}(u, v) q\right)\right] \geqslant 0
$$

or

$$
\sum_{i=1}^{k} \lambda_{i}\left(\eta^{\top}(x, u)\left[\nabla_{u} f_{i}(u, v)+t_{i}+\nabla_{u u} f_{i}(u, v) q\right]\right) \geqslant 0 .
$$

Since $\lambda \in \operatorname{int}\left(K^{*}\right)$, we have

$$
-\eta^{\top}(x, u)\left[\nabla_{u} f(u, v)+t+\nabla_{u u} f(u, v) q\right] \notin \operatorname{int}(K) .
$$

From hypothesis (i), we obtain

$$
\left[f(x, v)-f(u, v)+\frac{1}{2} q^{\top} \nabla_{u u} f(u, v) q\right] \in K
$$

and

$$
\left[x^{\top} t-u^{\top} t\right] \in K .
$$

Combining above inequalities, we get

$$
\left[f(x, v)+x^{\top} t-f(u, v)-u^{\top} t+\frac{1}{2} q^{\top} \nabla_{u u} f(u, v) q\right] \in K .
$$


From (3.6) and (3.7), we get

$$
\left[f(x, v)+x^{\top} t-f(u, v)-u^{\top} t+\frac{1}{2} q^{\top} \nabla_{u u} f(u, v) q\right] \in K .
$$

Similarly, using hypothesis (iii) and primal constraints, it follows that

$$
\xi^{\top}(v, y)\left[\nabla_{y} f(x, y)-r+\nabla_{y y} f(x, y) p\right] \notin \operatorname{int}(K) .
$$

Again, from hypothesis (ii), we obtain

$$
\left[-f(x, y)+y^{\top} r+f(x, v)-v^{\top} r+\frac{1}{2} p^{\top} \nabla_{y y} f(x, y) p\right] \notin \operatorname{int}(K) .
$$

Adding inequalities(3.4)-(3.8) and using the fact that $x^{\top} t \leqslant s(x \mid B)$ and $v^{\top} r \leqslant s(v \mid \mathrm{F})$, we have

$$
\left[-f(x, y)+y^{\top} r+f(x, v)-v^{\top} r+\frac{1}{2} p^{\top} \nabla_{y y} f(x, y) p\right] \in \operatorname{int}(K)
$$

which contradicts (3.9). Hence the result is obtained.

Theorem 3.2 (Weak duality theorem). Let $(x, y, \lambda, r, p) \in R^{0}$ and $(u, v, \lambda, t, q) \in S^{0}$. Let

(i) $\mathrm{f}(., v)$ be K-pseudobonvex and $(.)^{\top} \mathrm{t}$ be K-psedoinvex at $\mathrm{u}$ with respect to $\eta$;

(ii) $\mathrm{f}(\mathrm{x},$.$) be strongly \mathrm{K}-p$ seudoconcave and $(.)^{\mathrm{T}} \mathrm{r}$ be strongly $\mathrm{K}$ - psedoincave at $\mathrm{y}$ with respect to $\xi$;

(iii) $\eta(x, u)+u+q \in C_{1}$ for all $x \in C_{1}$;

(iv) $\xi(v, y)+y+p \in C_{2}$ for all $y \in C_{2}$.

Then

$$
T(u, v, t, q)-W(x, y, r, p) \notin \operatorname{int}(K) .
$$

Proof. The proof is clear from Theorem 3.1.

For proving the strong and converse duality results, it is convenient to rewrite the (NSOP) and (NSOD) in the given form.

$$
\begin{aligned}
& \text { (NSOP) K-Minimize } W(x, y, r, p)=\left(f_{1}(x, y)+s\left(x \mid B_{1}\right)-y^{\top} r_{1}-\frac{1}{2} p^{\top} \nabla_{y y} f_{1}(x, y) p, f_{2}(x, y)\right. \\
& +s\left(x \mid B_{2}\right)-y^{\top} r_{2}-\frac{1}{2} p^{\top} \nabla_{y y} f_{2}(x, y) p, \ldots, f_{k}(x, y)+s\left(x \mid B_{k}\right) \\
& \left.-y^{\top} r_{k}-\frac{1}{2} p^{\top} \nabla_{y y} f_{k}(x, y) p\right) \\
& \text { subject to } \left.\quad-\sum_{i=1}^{k} \lambda_{i}\left[\nabla_{y} f_{i}(x, y)-r_{i}+\nabla_{y y} f_{i}(x, y)\right\} p\right] \in C_{2}^{*} \text {, } \\
& y^{\top}\left[\sum_{i=1}^{k} \lambda_{i}\left(\nabla_{y} f_{i}(x, y)-r_{i}+\nabla_{y y} f_{i}(x, y) p\right)\right] \geqslant 0, \\
& p^{\top}\left[\sum_{i=1}^{k} \lambda_{i}\left(\nabla_{y} f_{i}(x, y)-r_{i}+\nabla_{y y} f_{i}(x, y) p\right)\right] \geqslant 0 \text {, } \\
& x \in C_{1}, r_{i} \in F_{i}(i=1,2, \ldots, k), \lambda \in \operatorname{int}\left(K^{*}\right),
\end{aligned}
$$

(NSOD) K-Maximize $T(u, v, t, q)=\left(f_{1}(u, v)-s\left(v \mid F_{1}\right)+u^{\top} t_{1}-\frac{1}{2} q^{\top} \nabla_{u u} f_{1}(u, v) q, f_{2}(u, v)\right.$ 


$$
\begin{aligned}
& \quad-s\left(v \mid F_{2}\right)+u^{\top} t_{2}-\frac{1}{2} q^{\top} \nabla_{u u} f_{2}(u, v) q, \ldots, f_{k}(u, v)-s\left(v \mid F_{k}\right) \\
& \left.+u^{\top} t_{k}-\frac{1}{2} q^{\top} \nabla_{u u} f_{k}(u, v) q\right), \\
& \text { subject to } \quad \sum_{i=1}^{k} \lambda_{i}\left[\nabla_{u} f_{i}(u, v)+t_{i}+\nabla_{u u} f_{i}(u, v) q\right] \in C_{1}^{*}, \\
& u^{\top}\left[\sum_{i=1}^{k} \lambda_{i}\left(\nabla_{u} f_{i}(u, v)+t_{i}+\nabla_{u u} f_{i}(u, v) q\right)\right] \leqslant 0, \\
& q^{\top}\left[\sum_{i=1}^{k} \lambda_{i}\left(\nabla_{u} f_{i}(u, v)+t_{i}+\nabla_{u u} f_{i}(u, v) q\right)\right] \leqslant 0, \\
& v \in C_{2}, t_{i} \in B_{i}(i=1,2, \ldots, k), \lambda \in \operatorname{int}\left(K^{*}\right) .
\end{aligned}
$$

Theorem 3.3 (Strong duality theorem). Let $(\bar{x}, \bar{y}, \bar{r}, \bar{p})$ be a weak minimum of (NSOP), and fix $\lambda=\bar{\lambda}$ in (NSOD). Assume that

(i) for every $(v, q) \in X \times R^{m}, v \in C_{2},\left\{\nabla_{u}\left(f_{i} \bar{u}, \bar{v}\right)+\bar{t}_{i}+\nabla_{u u} f_{i}(\bar{u}, \bar{v}) \bar{q}\right\}$ is linearly independent, for every $i=1,2, \ldots, k$;

(ii) $\left\{\nabla_{u u} f_{i}(\bar{u}, \bar{v})\right\}$ is nonsingular, for every $i=1,2, \ldots, k$;

(iii) if $\left.\overline{\mathrm{q}}^{\top}\left[\nabla_{\mathfrak{u}} \mathrm{f}_{\mathfrak{i}}(\overline{\mathrm{u}}, \bar{v})+\overline{\mathrm{t}}_{\mathfrak{i}}+\nabla_{\mathfrak{u} u} \mathrm{f}_{\mathfrak{i}}(\overline{\mathrm{u}}, \bar{v}) \overline{\mathrm{q}}\right\}\right]=0$, then $\overline{\mathrm{q}}=0$;

(iv) $R_{+}^{k} \subset K$.

Then, there exists $\overline{\mathrm{r}} \in \mathrm{F}$ such that $(\overline{\mathrm{x}}, \overline{\mathrm{y}}, \overline{\mathrm{r}}, \overline{\mathrm{p}})$ is a weak minimum for the problem (NSOD).

Proof. The proof of strong duality theorems follows almost same lines given by [7].

Remark 3.4. In case of symmetric programming problem, the proof remains the same as strong duality theorems.

Theorem 3.5 (Strict converse duality theorem). Let $(\overline{\mathrm{u}}, \overline{\mathrm{v}}, \overline{\mathrm{t}}, \overline{\mathrm{q}})$ be a weak minimum of (NSOD), and fix $\lambda=\bar{\lambda}$ in (NSOP). Assume that

(i) for every $(x, p) \in X \times R^{m}, y \in C_{2},\left\{\nabla_{y}\left(f_{i} \bar{x}, \bar{y}\right)-\bar{r}_{i}+\nabla_{y y} f_{i}(\bar{x}, \bar{y}) \bar{p}\right\}$ is linearly independent for every $i=1,2, \ldots, k$;

(ii) $\left\{\nabla_{y y} f_{i}(\bar{x}, \bar{y})\right\}$ is nonsingular, for every $i=1,2, \ldots, k$;

(iii) if $\left.\bar{p}^{\top}\left[\nabla_{y} f_{i}(\bar{x}, \bar{y})-\bar{r}_{i}+\nabla_{y y} f_{i}(\bar{x}, \bar{y}) \bar{p}\right\}\right]=0$, then $\bar{p}=0$;

(iv) $R_{+}^{k} \subset K$.

Then, there exists $\overline{\mathrm{t}} \in \mathrm{B}$ such that $(\overline{\mathrm{u}}, \overline{\mathrm{v}}, \overline{\mathrm{t}}, \overline{\mathrm{q}})$ is a weak minimum for the problem (NSOD).

Proof. The proof is obvious from Theorem 3.3.

\section{Acknowledgment}

The authors are thankful to the anonymous referees and editor for their valuable suggestions, which have substantially improved the presentation of the paper. The first author gratefully acknowledges the J. C. Bose University of Science and Technology, YMCA, Faridabad-121 006, India.

\section{References}

[1] X. H. Chen, Higher-order symmetric duality in nondifferentiable multiobjective programming problems, J. Math. Anal. Appl., 290 (2004), 423-435. 1

[2] R. Dubey, Deepmala, V. N. Mishra, Higher-order symmetric duality in nondifferentiable multiobjective fractional programming problem over cone contraints, Statist. Optim. Infor. Comput., 8 (2020), 187-205. 2.1

[3] R. Dubey, A. Kumar, R. Ali, L. N. Mishra, New class of G-Wolfe-type symmetric duality model and duality relations under $\mathrm{G}_{\mathrm{f}}$-bonvexity over arbitrary cones, J. Inequal. Appl., 30 (2020), 30 pages. 1 
[4] R. Dubey, V. N. Mishra, Symmetric duality results for second-order nondifferentiable multiobjective programming problem, RAIRO-Oper. Res., 53 (2019), 539-558.

[5] R. Dubey, L. N. Mishra, R. Ali, Special class of second-order non-differentiable symmetric duality problems with ( $\left.G, \alpha_{f}\right)$ pseudobonvexity assumptions, Mathematics, 763 (2019), 16 pages.

[6] R. Dubey, L. N. Mishra, L. M. S. Ruiz, Nondifferentiable G-Mond-Weir type multiobjective symmetric fractional problem and their duality theorems under generalized assumptions, Symmetry, 11 (2019), 18 pages. 1

[7] Y. Gao, Higher order symmetric duality in multiobjective programming problems, Acta Math. Appl. Sin. Engl. Ser., 32 (2016), 485-494. 3

[8] M. A. Hanson, Second-order invexity and duality in mathematical programming, Opsearch, 30 (1993), 313-320. 1

[9] A. Jayswal, I. M. Stancu-Minasian, D. Kumar, Higher-order duality for multiobjective programming problem involving (F, $\alpha, \rho, d)$-V-type-I functions, J. Math. Model. Algorithms Oper. Res., 13 (2014), 125-141. 1

[10] O. L. Mangasarian, Second and higher-order duality in nonlinear programming, J. Math. Anal. Appl., 51 (1975), 607620. 1

[11] M. V. Stefaneseu, M. Ferrara, A. Stefanescu, Multiobjective programming with new invexities, Optim. Lett., 7 (2013), 855-870. 1

[12] S. K. Suneja, S. Agarwal, S. Davar, Multiobjective symmetric duality involving cones, European J. Oper. Res., 141 (2002), 471-479. 2.7

[13] S. K. Suneja, M. K. Srivastava, M. Bhatia, Higher order Duality in Multiobjective Fractional Programming with Support Functions, J. Math. Anal. Appl., 347 (2008), 8-17. 1

[14] Vandana, R. Dubey, Deepmala, L. N. Mishra, V. N. Mishra, Duality relations for a class of a multiobjective fractional programming problem involving support functions, Amer. J. Oper. Res., 8 (2018), 294-311. 1

[15] G. Ying, Higher-order symmetric duality for a class of multiobjective fractional programming problems, J. Inequal. Appl., 2012 (2012), 12 pages. 1 